OPEN ACCESS

Edited by:

Alessia Libera Gazzonis,

University of Milan, Italy

Reviewed by:

Luca Villa,

University of Milan, Italy

Münir Aktaş,

Firat University, Turkey

*Correspondence:

Mourad Ben Said

bensaidmourad83@yahoo.fr

Lilia Messadi

lilia_messadi@yahoo.fr

tThese authors share last authorship

Specialty section:

This article was submitted to

Parasitology,

a section of the journal

Frontiers in Veterinary Science

Received: 06 March 2021

Accepted: 14 April 2021

Published: 26 May 2021

Citation:

Belkahia H, Selmi R, Zamiti S,

Daaloul-Jedidi M, Messadi $L$ and Ben Said M (2021) Zoonotic Rickettsia

Species in Small Ruminant Ticks From

Tunisia. Front. Vet. Sci. 8:676896.

doi: 10.3389/fvets.2021.676896

\section{Zoonotic Rickettsia Species in Small Ruminant Ticks From Tunisia}

\author{
Hanène Belkahia ${ }^{1}$, Rachid Selmi ${ }^{1,2}$, Sayed Zamiti ${ }^{1}$, Monia Daaloul-Jedidi ${ }^{1}$, Lilia Messadi ${ }^{\text {*t }}$ \\ and Mourad Ben Said ${ }^{1,3 * t}$ \\ ${ }^{1}$ Service de Microbiologie et Immunologie, Ecole Nationale de Médecine Vétérinaire, University of Manouba, Sidi Thabet, \\ Tunisia, ${ }^{2}$ Ministère de la Défense Nationale, Direction Générale de la Santé Militaire, Service Vétérinaire, Tunis, Tunisia, \\ ${ }^{3}$ Département des Sciences Fondamentales, Institut Supérieur de Biotechnologie de Sidi Thabet, University of Manouba, \\ Sidi Thabet, Tunisia
}

Tick-borne rickettsioses present a significant public health threat among emerging tick-borne diseases. In Tunisia, little is known about tick-borne Rickettsia pathogens. Therefore, the aim of this study was to investigate the presence of Rickettsia species in small ruminant ticks from Tunisia. Adult ticks $(n=694)$ were collected from goats and sheep in northern Tunisia. Obtained ticks were identified as Rhipicephalus turanicus ( $n=434)$ and Rhipicephalus sanguineus sensu lato $(n=260)$. Selected ticks $(n=666)$ were screened for the presence of Rickettsia spp. by PCR targeting a partial sequence of the ompB gene followed by sequence analysis. Rickettsial DNA was detected in 122 (18.3\%) tested tick samples. The infection rates in Rh. turanicus and $R h$. sanguineus s.l. ticks were 23.4 and 9.5\%, respectively. The overall prevalence of rickettsial DNA was markedly higher in ticks collected from goats (23.2\%) compared to those infesting sheep (7.9\%). The detection of rickettsial DNA was significantly higher in ticks from the governorate of Beja (39.0\%) than those from the governorate of Bizerte (13.9\%). Two additional genes, the outer membrane protein A gene (ompA) and the citrate synthase gene ( $g / t A)$, were also targeted for further characterization of the detected Rickettsia species. Genotyping and phylogenetic analysis based on partial sequences $(n=106)$ of the three different genes revealed that positive ticks are infected with different isolates of two Spotted Fever Group (SFG) Rickettsia, namely, Rickettsia massiliae and Rickettsia monacensis, closely related to those infecting camels and associated ticks from Tunisia, and humans and small ruminant ticks from neighboring countries like Italy, France, and Spain.

Keywords: Rickettsia species, Rhipicephalus ticks, molecular survey, genotyping, phylogenetic analysis, Tunisia

\section{INTRODUCTION}

Rickettsia species (family Rickettsiaceae; order Rickettsiales) are included into four groups: the spotted fever group (SFG) rickettsiae, the typhus group, the Rickettsia bellii group, and the Rickettsia canadensis group (1). These pathogens infected several domesticated and wild vertebrate hosts through hematophagous arthropod vectors bites (mainly ticks, fleas, and mites). Besides, tick-borne rickettsioses are considered as one of the most virulent zoonotic diseases affecting humans especially in African countries (2). 
Spotted fever group rickettsioses (SFG) are actually considered as emerging and reemerging diseases affecting animals worldwide. They are caused by the pathogenic and zoonotic spotted fever Rickettsia bacteria mainly transmitted by ticks. Humans may be accidently infected especially in tropical areas $(1,2)$.

In Tunisia, several SFG Rickettsia species have been previously reported, as Rickettsia conorii, that was described for the first time in humans since 1910 (3), and, recently, by Znazen et al. (4) and Khrouf et al. (5). In addition, $R$. conorii subsp. israelensis was identified in one human and tick specimens of Rhipicephalus sanguineus s.l. complex collected from dogs $(4,6)$. Furthermore, $R$. aeschlimannii, $R$. helvetica, and $R$. africae were reported from camels' blood samples and infesting Hyalomma tick tissues in southern and central Tunisia $(7,8)$. DNA of $R$. helvetica was also identified in questing Ixodes ricinus ticks (9).

Rickettsia massiliae and Rickettsia monacensis, belonging to the SFG rickettsiae, are widely identified among animals, humans, and arthropod vectors (1). Rickettsia massiliae was firstly isolated in France from Rhipicephalus turanicus tick (10). Since then, this pathogen has been transmitted by and/or isolated from Rhipicephalus ticks like $R h$. turanicus, $R h$. sanguineus sensu lato (s.l.), Rh. bursa, and Rh. pusillus collected from domestic and wild animals such as cattle, goats, horses, dogs, cats, hedgehogs, red foxes, and hares in different worldwide countries (11-16). In Tunisia, $R$. massiliae was previously detected in $R h$. sanguineus s.l. ticks collected from dogs (6), in peripheral blood of camels (8), and in skin biopsy of one patient (5). Interestingly, this bacterium is recognized as pathogenic in human and may be clinically expressed as a febrile illness with maculopapular rash, fever, night sweats, headache, and necrotic eschar at the tick bite site $(17,18)$.

Rickettsia monacensis was earlier detected in I. ricinus ticks from several European countries like Italy, Spain, Romania, Bulgaria, Hungary, and Serbia $(1,12)$. In our country, the first identification of $R$. monacensis was also reported in $I$. ricinus ticks by Sfar et al. (9). Additionally, this human-pathogenic species was recently detected not only in Tunisian camels but also in associated $H$. impeltatum ticks removed from uninfected animals (8). This bacterium causes from moderate to severe infections in humans including fever, rash on palms and soles, and inoculation eschar $(19,20)$. To better understand the epidemiology of Rickettsia species in Tunisia, we investigated, in the present molecular survey the occurrence of rickettsial bacteria in small ruminant ticks according to potential risk factors. Molecular characterization and phylogenetic analysis of revealed Rickettsia spp. isolates were also performed by using three different gene fragments.

\section{MATERIALS AND METHODS}

\section{Study Area Description}

A cross-sectional study was carried out in five localities of Northern Tunisia (Figure 1). El Alia $37^{\circ} 16^{\prime} \mathrm{N} ; 10^{\circ} 03^{\prime} \mathrm{E}$ and Khetmine $37^{\circ} 16^{\prime} \mathrm{N} ; 9^{\circ} 99^{\prime} \mathrm{E}$ fall in the sub-humid bioclimatic zone with an average annual rainfall of $400 \mathrm{~mm}$ and a mean temperature of $18.4^{\circ} \mathrm{C}$ while Joumine $36^{\circ} 92^{\prime} \mathrm{N}$; $9^{\circ} 38^{\prime} \mathrm{E}$, Sejnane $37^{\circ} 15^{\prime} \mathrm{N} ; 9^{\circ} 23^{\prime} \mathrm{E}$, and Amdoun $36^{\circ} 76^{\prime} \mathrm{N}$; $9^{\circ} 08^{\prime} \mathrm{E}$ are characterized by humid climate with an average annual rainfall of $650 \mathrm{~mm}$ and a mean temperature of $14.4^{\circ} \mathrm{C}$.

\section{Tick Collection and Identification}

Ticks were collected from 303 apparently healthy goats (233 doe and 70 buck) and 160 healthy sheep (110 ewes and 50 rams). Goats were originated from 16 herds located in Sejnane $(N=3)$, El Alia $(N=4)$, and Joumine $(N=5)$ belonging to the Bizerte governorate and in Amdoun ( $N=4$, Beja governorate). Sheep derived from nine herds from El Alia $(N=4)$ and Khetmine $(N=5)$ in the governorate of Bizerte.

All partially engorged ticks were collected by using a clamp from different preferred sites of small ruminant body (ears, neck, udder, and external genitalia) and separately categorized according to the examined animal host. Obtained specimens were morphologically identified using the taxonomic key of Walker et al. (21) and then classified according to tick species, life stage, and gender. Each tick specimen was individually conserved in a tube containing $70 \%$ ethanol and stored at $-20^{\circ} \mathrm{C}$.

\section{Total DNA Extraction and Tick DNA Amplification}

Each identified tick was washed with sterile water, dried, and crushed individually using an automated TissueLyser LT system (Qiagen, Hilden, Germany). Genomic DNA extraction was performed from each tick sample using the DNeasy tissue kit (Qiagen, Hilden, Germany). Obtained DNA extracts were stored at $-20^{\circ} \mathrm{C}$. DNA extraction efficiency was validated by PCR amplification step targeting the ribosomal RNA subunit (16S rRNA) gene using the tick-specific primers TQ16S+1F and TQ16S-2R as described by Black and Piesman (22) (Table 1).

\section{Molecular Detection of Rickettsia spp.}

In order to identify all species of the Rickettsia genus, tick DNA samples were subjected to nested PCR targeting a fragment (425 bp) of the rickettsial outer membrane protein B (ompB) gene (23) (Table 1). For further characterization, the outer membrane protein $\mathrm{A}(\operatorname{ompA})$ and the citrate synthase protein (gltA) gene fragments (532 and 381 bp, respectively) were amplified by using nested and endpoint PCR, respectively (Table 1). PCR reactions were performed in an automated DNA thermal cycler. Thermal cycling profiles were as described by Oteo et al. (24), and Regnery et al. (25), respectively.

The PCR reactions were carried out in a final volume of 50 $\mu l$ composed of $0.125 \mathrm{U} / \mu \mathrm{L}$ of Taq DNA polymerase (Biobasic Inc., Markham, Canada), $1 \times$ PCR buffer, $1.5 \mathrm{mM} \mathrm{MgCl} 2,0.2 \mathrm{mM}$ of dNTP, $3 \mu \mathrm{L}$ of genomic DNA (50-150 ng) in the first PCR and $1 \mu \mathrm{L}$ in the second PCR (for nested PCR), $0.5 \mu \mathrm{M}$ of the primers, and autoclaved water. PCR products were visualized using electrophoresis in $1.5 \%$ agarose gels stained with ethidium bromide and observed under UV transillumination.

\section{Statistical Analysis}

Exact confidence intervals (CI) at the 95\% level were estimated for prevalence rates according to different considered factors. 


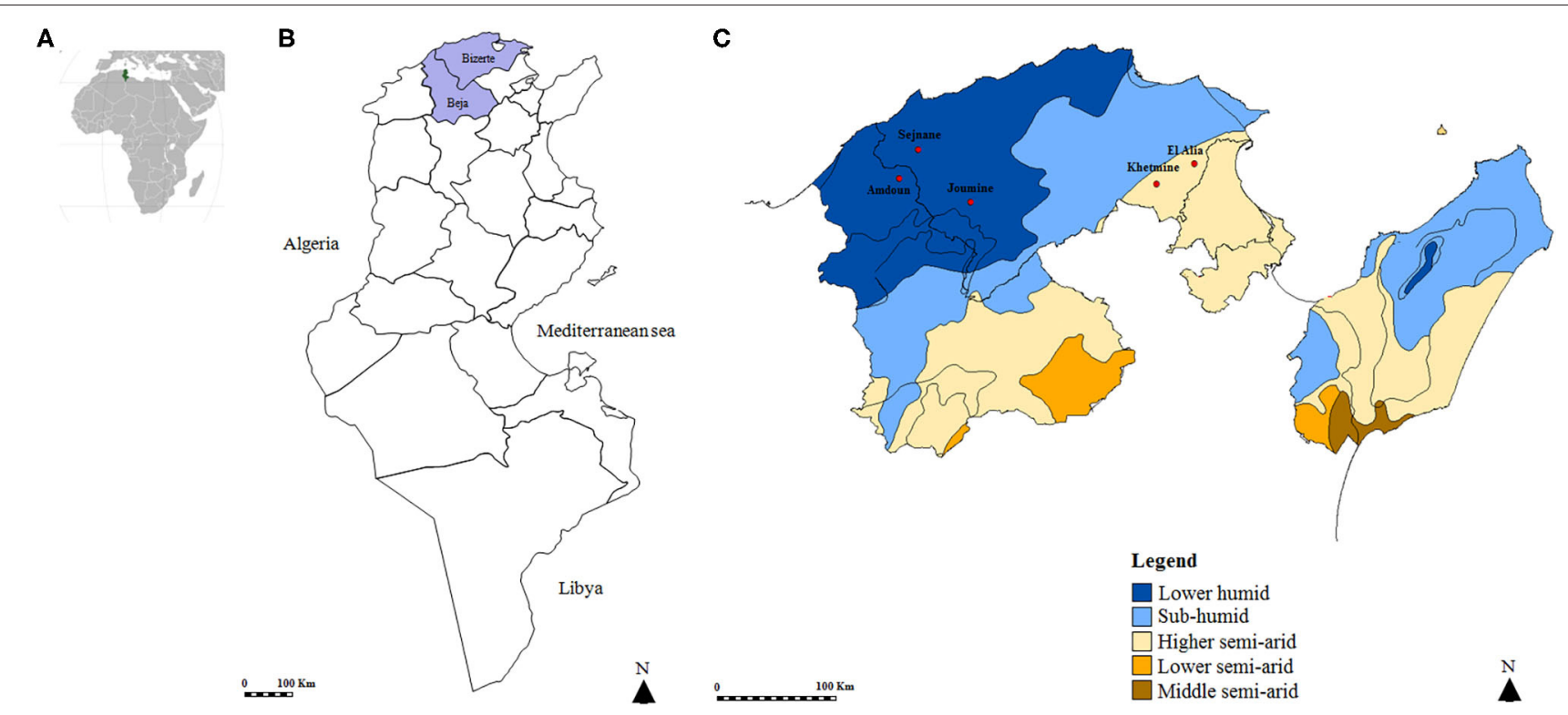

FIGURE 1 | Map of the Tunisian studied regions. (A) Geographical position of Tunisia in the African continent. (B) Map of Tunisia showing investigated governorates. (C) Position of studied localities according to bioclimatic areas.

TABLE 1 | Primers used for the identification and/or genetic characterization of Rickettsia species infecting ticks collected in this study from small ruminants.

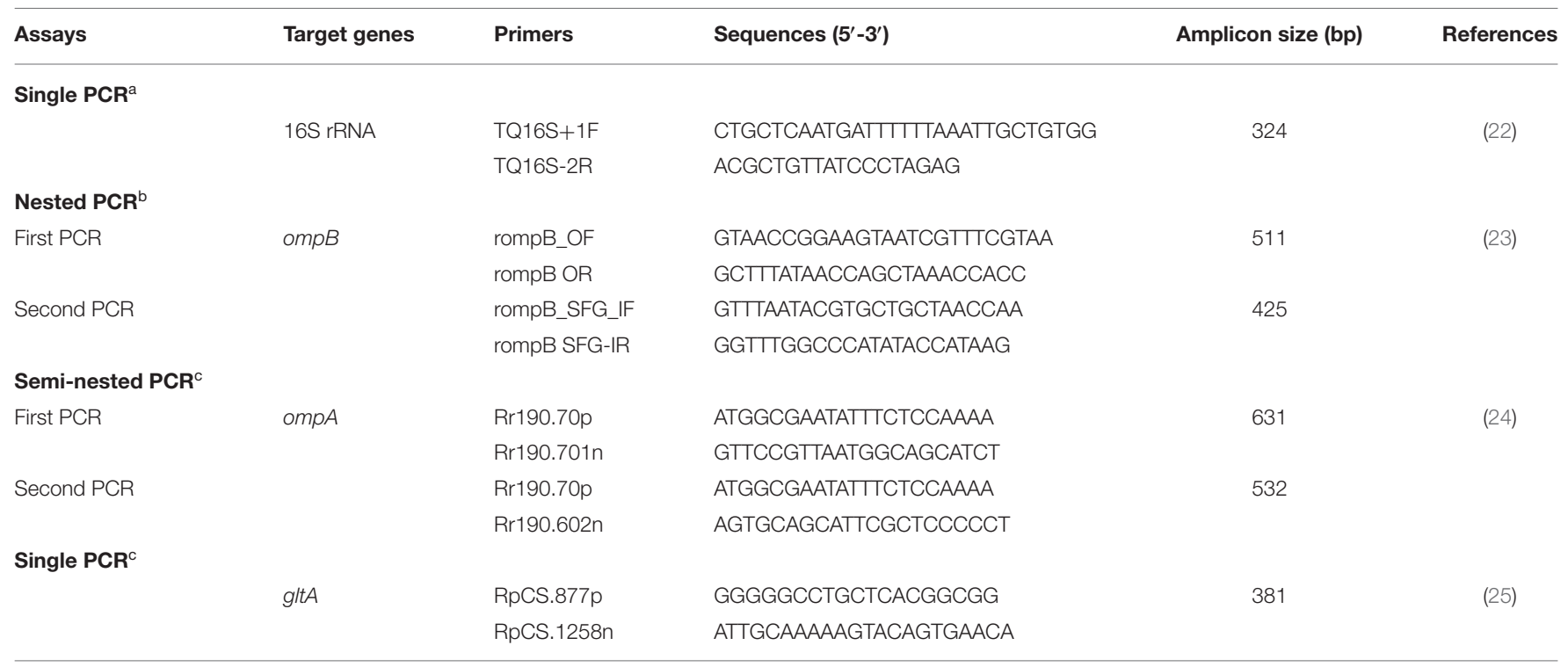

a Single PCR based on the 16S rRNA gene allowing the selection of tick samples with DNA extraction efficiency.

${ }^{b}$ Nested PCR based on the OMPB gene allowing the detection and/or characterization after sequencing of Rickettsia species.

' Single and semi-nested PCR based on gltA and ompA genes, respectively, allowing the characterization after sequencing of Rickettsia species.

A comparison of the prevalence of Rickettsia species in ticks according to abiotic factors (geographic location and bioclimatic conditions) and factors related to ticks (gender, age, and host origin) was carried out using the Epi Info 6 software 01 (CDC, Atlanta, USA) and the $\chi^{2}$-test. A difference is considered statistically significant when the degree of significance $p$ is $\leq 0.05$. In order to assess possible confusion between the risk factors, a Mantel-Haenszel $\chi^{2}$-test was performed.

\section{DNA Sequencing and Obtaining Final Sequences}

A total of 106 positive PCR products obtained after ompB, $o m p A$, and gltA PCRs were randomly selected and purified using the GF-1 Ambi Clean kit (Vivantis, USA), according to the manufacturer's instructions. Purified DNA amplicons were sequenced in both directions, using the same primers as for the single gltA PCR and the second PCR of each 
nested PCR amplification targeting ompA and $о т p B$ genes. The Big Dye Terminator cycle sequencing ready reaction kit (Applied Biosystems, Foster City, USA) and an ABI3730XL automated DNA sequencer (Macrogen Europe, Amsterdam, The Netherlands) were employed.

The chromatograms were evaluated with Chromas Lite $\mathrm{v}$ 2.01 (http://www.technelysium.com.au/chromas_lite.html). To obtain maximal data accuracy, sequences were determined on both forward and reverse strands. Indeed, the complementary strands of each sequenced product were manually assembled by using the DNAMAN software (Version 5.2.2; Lynnon Biosoft, Que., Canada). The primer region sequences were automatically removed and the overlapping parts were selected.

\section{Sequence Alignment and Phylogenetic Study}

Multiple-sequence alignments and sequence similarities were calculated using the CLUSTAL W method (26). BLAST analysis was performed to assess the level of similarity with previously reported sequences (http://blast.ncbi.nlm.nih.gov/). By using the DNAMAN software, genetic distances among the operational taxonomic units were computed by the maximum composite likelihood method (27) and were used to construct neighborjoining trees (28). Statistical support for internal branches of trees was evaluated by bootstrapping with 1,000 iterations (29).

\section{RESULTS}

\section{Tick Species Recognition}

A total of 694 ticks were collected from goats (460/694, $66.3 \%)$ and sheep $(234 / 694,33.7 \%)$ from a higher semiarid area $(374 / 694,53.9 \%)$ and a low humid area (320/694, 46.1\%). Almost all specimens were removed from animals located in the governorate of Bizerte (82\%) while ticks collected from small ruminants in El Alia were the most numerous (43.5\%) compared to those in other localities (Figure 1 and Table 2). The sex ratio of ticks collected from these animals (M/F) was 1.14. The intensity of tick infestation is estimated at 1.52 and 1.46 ticks/animal for goats and sheep, respectively. Two tick species belonging to Rhipicephalus genus were identified, namely, Rh. turanicus $(434 / 694,62.5 \%)$ and $R h$. sanguineus s.l. (260/694, $37.5 \%$ ) (Table 2).

\section{Efficiency of DNA Isolation}

DNA extracts were tested and validated in 666 samples (96\%). No amplification products were obtained for 28 samples, reflecting a probable failure of the DNA extraction, and were thus excluded from the analysis. Thereby, a total of 666 ticks were selected from goats $(452 / 666,67.9 \%)$ and sheep $(214 / 666,32.1 \%)$ from the higher semiarid area $(357 / 666,53.6 \%)$ and the low humid area $(309 / 666,46.4 \%)$. Almost all analyzed ticks were collected from small ruminants located in the governorate of Bizerte $(82.3 \%)$ while ticks collected from animals in El Alia are the most numerous (43.4\%) compared to those in other localities (Figure 1 and Table 2). The sex ratio of tested ticks $(\mathrm{M} / \mathrm{F})$ was 1.15 . After the validation of DNA extracts, a total of $423 \mathrm{Rh}$. turanicus
(63.5\%) and 243 Rh. sanguineus s.l. (36.5\%) were subjected to Rickettsia spp. screening (Table 2).

\section{Rickettsia spp. Screening and Risk Factor Analysis}

Based on ompB gene analysis, DNA of Rickettsia spp. was identified in 122 tick samples (18.3\%) (Table 2). Infection among $R$ h. turanicus ticks is statistically more prevalent $(23.4 \%)$ compared to Rh. sanguineus s.l. (9.5\%) ( $p<0.001)$. Ticks collected from goats were statistically more infected with Rickettsia spp. (23.2\%) than those from sheep (7.9\%) ( $p<$ 0.001; Table 2). Ticks removed from small ruminants located in the governorate of Beja were statistically more infected with Rickettsia spp. (39.0\%) $(p<0.001)$ than those in the governorate of Bizerte (13.9\%) $(p<0.001)$. Specimens from Amdoun (39.0\%) and El Alia (24.6\%) localities were more infected with Rickettsia spp. than those from Sejnane $(3.0 \%)$, Khetmine (1.5\%), and Joumine $(0 \%)(p<0.001$; Table 2). In contrast, no statistically significant differences in Rickettsia spp. infection rates were observed according to tick gender and bioclimatic areas $(p<0.05$, Table 2$)$.

\section{Identification of Rickettsia Species Infecting Ticks}

Two rickettsial species were identified in small ruminants' ticks, namely, R. massiliae and R. monacensis (Table 3). Based on ompB gene analysis, 40 PCR products ( 32 from $R h$. turanicus and eight from $R h$. sanguineus s.l.) were sequenced successfully. Rickettsia massiliae was identified in $R h$. turanicus $(n=32,100 \%)$ and $R h$. sanguineus s.l. ( $n=6,75 \%)$. However, $R$. monacensis DNA was found in Rh. sanguineus s.l. $(n=2,25 \%)$. Based on ompA and gltA gene analysis, PCR products were sequenced successfully from 41 and 25 positives samples, respectively. Rickettsia massiliae was detected in $R h$. turanicus $(n=31,100 \%)$ and $R h$. sanguineus s.l. $(n=10,100 \%)$ based on ompA partial sequence analysis. However, using the gltA gene, DNA of this bacterium was found in $R$. turanicus $(n=25,100 \%)$ (Table 3 ).

\section{Molecular Characterization and Phylogenetic Analysis}

Out of 122 Rickettsia-positive samples, 94 gave a clear band in the correct nucleotide size of the partial genes $(o m p A, o m p B$, and $g l t A$ ) in at least one of the three genotyping PCRs. Partial sequences $(n=106)$ of the three analyzed genes were deposited under GenBank accession numbers presented in Table 4. Based on all revealed sequences of the three analyzed genes, we precisely selected Rickettsia spp. genotypes according to infecting tick species, and they differ from each other by at least one mutation in the nucleotidic sequence.

\section{Rickettsia spp. ompB Genotypes}

Rickettsia infection was confirmed by sequencing of 382-bp ompB fragments from randomly selected $32 R h$. turanicus- and eight $R$ h. sanguineus s.l. Rickettsia-positive samples (Tables 3, 4). Alignment of these sequences revealed two $R$. massiliae genotypes from $R h$. sanguineus s.l. (ompBRmasRs1 and ompBRmasRs2; GenBank accession numbers MN311185 and MN311189, 
TABLE 2 | Molecular prevalences of Rickettsia spp. according to tick species, tick gender, infested host, bioclimatic zone, governorate, and locality.

\begin{tabular}{|c|c|c|c|c|}
\hline Factors & Number of collected ticks $(\%)^{a}$ & Number of analyzed ticks $(\%)^{\mathrm{b}}$ & Positive $^{c}\left(\% \pm\right.$ C.I. $\left.{ }^{d}\right)$ & $P$-value (Khi2) \\
\hline Tick species & & & & $0.000^{*}(20.02)$ \\
\hline Rh. turanicus & $434(62.5)$ & $423(63.5)$ & $99(23.4 \pm 0.04)$ & \\
\hline Rh. sanguineus s.l. & $260(37.5)$ & $243(36.5)$ & $23(9.5 \pm 0.04)$ & \\
\hline Tick gender & & & & $0.519(0.42)$ \\
\hline Male & $370(53.3)$ & $356(53.4)$ & $62(17.4 \pm 0.04)$ & \\
\hline Female & $324(46.7)$ & $310(46.6)$ & $60(19.4 \pm 0.04)$ & \\
\hline Infested host & & & & $0.000^{\star}(22.65)$ \\
\hline Goats & $460(66.3)$ & $452(67.9)$ & $105(23.2 \pm 0.04)$ & \\
\hline Sheep & $234(33.7)$ & $214(32.1)$ & $17(7.9 \pm 0.03)$ & \\
\hline Bioclimatic zone & & & & $0.185(1.76)$ \\
\hline Higher semi-arid & $374(53.9)$ & $357(53.6)$ & $72(20.2 \pm 0.04)$ & \\
\hline Lower humid & $320(46.1)$ & $309(46.4)$ & $50(16.2 \pm 0.04)$ & \\
\hline Governorate & & & & $0.000^{\star}(40.87)$ \\
\hline Bizerte & $569(82.0)$ & $548(82.3)$ & $76(13.9 \pm 0.03)$ & \\
\hline Beja & $125(18.0)$ & $118(17.7)$ & $46(39.0 \pm 0.09)$ & \\
\hline Locality & & & & $0.000^{*}(87.96)$ \\
\hline El Alia & 302 (43.5) & $289(43.4)$ & $71(24.6 \pm 0.05)$ & \\
\hline Khetmine & $72(10.4)$ & $68(10.2)$ & $1(1.5 \pm 0.03)$ & \\
\hline Sejnane & $137(19.7)$ & $133(20.0)$ & $4(3.0 \pm 0.03)$ & \\
\hline Amdoun & $125(18.0)$ & $118(17.7)$ & $46(39.0 \pm 0.09)$ & \\
\hline Joumine & $58(8.4)$ & $58(8.7)$ & $0(0)$ & \\
\hline Total & $694(100)$ & $666(100)$ & $122(18.3 \pm 0.03)$ & \\
\hline
\end{tabular}

Rh. Turanicus, Rhipicephalus turanicus, Rh. sanguineus s.l., Rhipicephalus sanguineus sensu lato.

a Number of collected ticks submitted to PCR performed for the confirmation of the DNA extraction efficiency.

${ }^{b}$ Number of included ticks for Rickettsia spp. survey selected after the confirmation of the DNA extraction efficiency.

${ }^{c}$ Ticks positive to Rickettsia spp. according to the total number of analyzed ticks.

${ }^{d}$ C.I.: $95 \%$ confidence interval.

*Statistically significant test.

TABLE 3 | Rickettsia species identified by the sequencing of ompB, ompA, and gltA partial sequences in Rhipicephalus ticks.

\begin{tabular}{|c|c|c|c|c|c|c|}
\hline Tick species & Number & $\begin{array}{l}\text { ompB PCR } \\
\text { positive (\%) }\end{array}$ & $\begin{array}{c}\text { ompB PCR } \\
\text { positives/sequencing }\end{array}$ & $\begin{array}{c}\text { ompA PCR } \\
\text { positives/sequencing }\end{array}$ & $\begin{array}{c}\text { gltA PCR } \\
\text { positives/sequencing }\end{array}$ & Rickettsia spp. \\
\hline \multirow[t]{2}{*}{ Rh. turanicus } & 423 & $99(23.4 \pm 0.04)$ & 32 & 31 & 25 & R. massiliae \\
\hline & & & 0 & 0 & 0 & R. monacensis \\
\hline \multirow[t]{2}{*}{ Rh. sanguineus s.I. } & 243 & $23(9.5 \pm 0.04)$ & 6 & 10 & 0 & R. massiliae \\
\hline & & & 2 & 0 & 0 & R. monacensis \\
\hline
\end{tabular}

Rh. turanicus, Rhipicephalus turanicus; Rh. sanguineus s.l., Rhipicephalus sanguineus sensu lato.

respectively) and two $R$. massiliae genotypes from $R h$. turanicus ticks (ompBRmasRt1 and ompBRmasRt2; GenBank accession numbers MN311191 and MN311211, respectively) (Table 4). In addition, two $R$. monacensis genotypes from $R h$. sanguineus s.l. (ompBRmonRs1 and ompBRmonRs2; GenBank Accession Numbers MN311223 and MN311224, respectively) were also recorded (Table 4 ).

A phylogenetic analysis based on the alignment of Tunisian genotypes with 31 Rickettsia spp. ompB sequences obtained from GenBank shows the assignment of revealed genotypes to $R$. massiliae and $R$. monacensis clusters. The $R$. massiliae cluster is formed by three subclusters supported by robustness node rates $\geq$ to $81 \%$ (Figure 2 ). Tunisian strains were assigned to the first and third subclusters. Genotypes ompBRmasRs2 and ompBRmasRt 2 were assigned to the first subcluster and clustered with strains isolated from $H$. impeltatum infesting camels in Tunisia and from Rh. sanguineus s.l. ticks located in Mediterranean countries such as Italy and Spain (Figure 2). Genotypes ompBRmasRs1 and ompBRmasRt1 were assigned to the third subcluster and clustered with strains isolated from $R h$. sanguineus s.l. and Rh. turanicus ticks originated from NorthMediterranean countries (Figure 2). The $R$. monacensis cluster is also formed by three subclusters supported by robustness rates of nodes $\geq$ to $81 \%$ (Figure 2). Genotypes ompBRmonRs 1 
TABLE 4 | Designation and information about sequencing of Rickettsia spp. genotypes identified in this study.

\begin{tabular}{|c|c|c|c|c|c|c|c|}
\hline Gene & Rickettsia sp. & Genotype & Number ${ }^{a}$ & Potential vector & Location $^{\mathrm{b}}$ & GenBank $^{c}$ & BLAST analysis \\
\hline \multirow[t]{6}{*}{ ompB } & R. massiliae & ompBRmasRs1 & 4 & Rh. sanguineus s.l. & Bizerte & MN311185 & 100\% R. massiliae (CP000683) \\
\hline & & ompBRmasRs2 & 2 & Rh. sanguineus s.l. & Bizerte & MN311189 & 100\% R. massiliae (KJ663751) \\
\hline & & ompBRmasRt1 & 20 & Rh. turanicus & Bizerte and Beja & MN311191 & 100\% R. massiliae (CP000683) \\
\hline & & ompBRmasRt2 & 12 & Rh. turanicus & Bizerte and Beja & MN311211 & 100\% R. massiliae (KJ663751) \\
\hline & R. monacensis & ompBRmonRs1 & 1 & Rh. sanguineus s.l. & Bizerte & MN311223 & 100\% R. monacensis (EU883092) \\
\hline & & ompBRmonRs2 & 1 & Rh. sanguineus s.l. & Bizerte & MN311224 & 99.4\% R. monacensis (EU883092) \\
\hline \multirow[t]{11}{*}{ ompA } & R. massiliae & ompARmasRs1 & 6 & Rh. sanguineus s.l. & Bizerte & MN311225 & 100\% R. massiliae (MH532237) \\
\hline & & ompARmasRs2 & 2 & Rh. sanguineus s.l. & Bizerte & MN311229 & 100\% R. massiliae (KJ663747) \\
\hline & & ompARmasRs3 & 1 & Rh. sanguineus s.l. & Beja & MW026194 & 99.8\% R. massiliae (MH532237) \\
\hline & & ompARmasRs4 & 1 & Rh. sanguineus s.l. & Beja & MW026195 & 99.8\% R. massiliae (MH532237) \\
\hline & & ompARmasRt1 & 16 & Rh. turanicus & Beja & MN311231 & 100\% R. massiliae (MH532237) \\
\hline & & ompARmasRt2 & 4 & Rh. turanicus & Beja & MW026200 & 100\% R. massiliae (KJ663747) \\
\hline & & ompARmasRt3 & 5 & Rh. turanicus & Beja & MW026204 & 99.8\% R. massiliae (MH532237) \\
\hline & & ompARmasRt4 & 2 & Rh. turanicus & Beja & MW026209 & 99.8\% R. massiliae (MH532237) \\
\hline & & ompARmasRt5 & 2 & Rh. turanicus & Beja & MW026211 & 99.6\% R. massiliae (MH532237) \\
\hline & & ompARmasRt6 & 1 & Rh. turanicus & Beja & MW026213 & 99.8\% R. massiliae (MH532237) \\
\hline & & ompARmasRt7 & 1 & Rh. turanicus & Bizerte & MW026214 & 99.6\% R. massiliae (KJ663747) \\
\hline gltA & R. massiliae & gltARmasRt1 & 25 & Rh. turanicus & Bizerte and Beja & MW026215 & 100\% R. massiliae (KJ663740) \\
\hline
\end{tabular}

R. sanguineus s.l., Rhipicephalus sanguineus sensu lato; R. turanicus, Rhipicephalus turanicus.

a Number of sequenced Rickettsia positive samples.

${ }^{b}$ Geographical location.

${ }^{c}$ GenBank accession number.

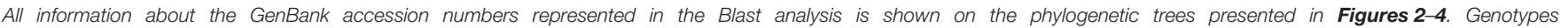

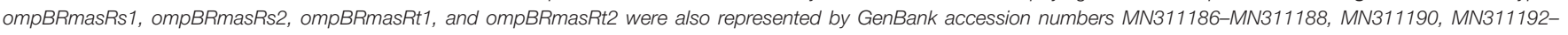

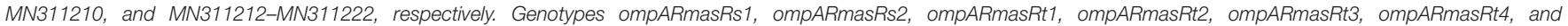



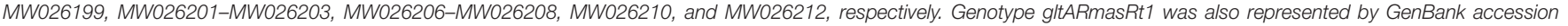
numbers MW026216-MW026239.

and ompBRmonRs2 were assigned, respectively, to the first and second subclusters. Genotype ompBRmonRs1 was closely related to isolates found in Tunisian camels and their infesting $H$. impeltatum ticks, and strains infecting human and ticks from different countries (Figure 2).

\section{Rickettsia spp. ompA Genotypes}

By using the ompA partial sequence, the infection with $R$. massiliae was revealed by sequencing of $490 \mathrm{bp}$ of the ompA gene from selected $31 \mathrm{Rh}$. turanicus- and $10 \mathrm{Rh}$. sanguineus s.l. Rickettsia-positive samples (Tables 3, 4). Alignment of these sequences confirmed the occurrence of four distinct genotypes from $R h$. sanguineus s.l. ticks (ompARmasRs1 to ompARmasRs4; GenBank Accession Numbers MN311225, MN311229 MW026194, and MW026195, respectively) and seven genotypes from $R h$. turanicus ticks (ompARmasRt1 to ompARmasRt7; GenBank Accession Numbers MN311231, MW026200, MW026204, MW026209, MW026211, MW026213, and MW026214, respectively) (Table 4 ).

For this gene, a phylogenetic tree based on the alignment of ompA partial sequences of Rickettsia spp. found in GenBank showed the presence of our sequences in the three subclusters that formed the R. massiliae cluster and supported by robustness node rates $\geq$ to $84 \%$ (Figure 3 ). Genotype ompARmasRt7 formed separately subcluster 1, and genotypes ompARmasRt2 and ompARmasRs2 were assigned to the last subcluster and clustered with strains isolated from $R h$. sanguineus s.l. located in different worldwide countries such as Italy, Austria, Argentina, and the USA. The remaining genotypes were clustered together in the second subcluster with several isolates infecting ticks from China and European countries (Figure 3).

\section{Rickettsia spp. gltA Genotypes}

Sequencing of $341 \mathrm{bp}$ of the gltA partial sequence obtained from 25 specimens of $R h$. turanicus-positive to Rickettsia spp. confirmed the infection with only one genotype (gltARmasRt1, GenBank accession number KJ663740) of $R$. massiliae (Tables 3, 4). This revealed that the genotype was $100 \%$ identical to strain $60 \mathrm{~B}$ infecting $R$ h. sanguineus s.l. tick collected from Italian human (GenBank Accession Number KJ663740) (Table 4).

Phylogenetic tree based on the gltA gene revealed that the gltARmasRt1 genotype clustered in the $R$. massiliae cluster especially in the first subcluster 1 with strains infecting Rh. sanguineus s.l. ticks from Italy and Argentina, Hyalomma asiaticum ticks from China, and $R$. turanicus tick specimens collected from birds in Portugal (Figure 4). 

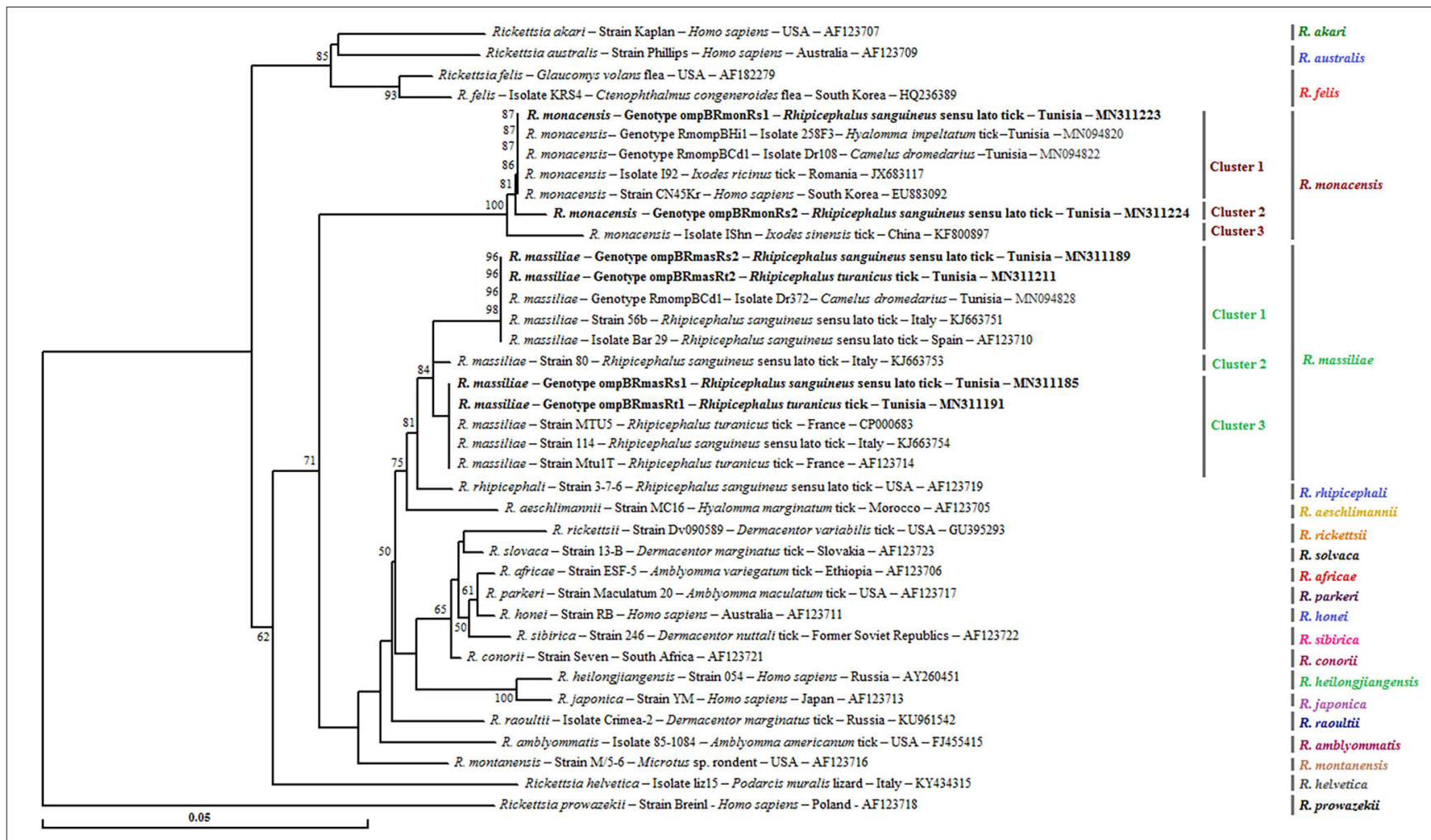

FIGURE 2 | Neighbor-joining tree based on the alignment of partial ompB sequences (382 bp) of Rickettsia spp. obtained in this study with selected sequences representative of the Rickettsia genus. Numbers over the branches indicate the percentage of replicated trees in which the associated taxa clustered together in the bootstrap test (1,000 replicates, only percentages $>50 \%$ were represented). The six partial ompB sequences representative of different Rickettsia spp. genotypes obtained in this study are indicated in bold. The host or vector, the genotype, strain or isolate name, the country of origin, and the GenBank accession number are indicated. One R. prowazekii ompB partial sequence was added as an outgroup.

\section{DISCUSSION}

Data about the occurrence and the genetic diversity of Rickettsia species in ticks is limited in North African countries (30,31), especially in Tunisia $(6,8,9)$. In this report, adult ticks infesting small ruminants in northern Tunisia were examined and two species of Rhipicephalus genus ( $R$. turanicus and $R$. sanguineus s.l.) were identified. This result is in agreement with other surveys which considered these two tick species as major ectoparasites of small ruminants in Tunisia $(32,33)$.

To our knowledge, we report here for the first time the detection of SFG Rickettsia DNA in ticks collected from small ruminants raised in the north of Tunisia. Although this study does not conclude on the competence of these potential vectors, given that these results do not suggest that the tick species mentioned in this report can serve as a competent vector for detected bacteria, this study made a contribution to the knowledge of the presence of SFG rickettsiae in Tunisia. In addition, present data showed the need to search these bacteria in animal hosts and to increase the investigated areas, the potentially incriminated risk factors, and the number of analyzed tick samples, including questing ticks and different life stages. All these information may facilitate future prevention against SFG Rickettsial diseases in the country.
Specifically, the detection of Rickettsia spp. DNA in $R h$. turanicus (23.4\%) and Rh. sanguineus s.l. (9.5\%) provides evidence that these tick species may be among the main vectors of Rickettsia species in northern Tunisia. These results are consistent with those reported by Khrouf et al. (6) who suggested a possible incrimination of Rhipicephalus ticks infesting dogs and sheep in the transmission of Rickettsia species in central Tunisia. Furthermore, according to Psaroulaki et al. (34), Rickettsia spp. were detected in Rhipicephalus ticks collected from domestic animals in Greece. Additionally, Germanakis et al. (35) reported that $R h$. turanicus has been implicated as a potential vector transmitting to humans several pathogens including Rickettsia species. In the Northwest of China, Wei et al. (36) suggested that $R$. massiliae, $R$. aeschlimannii, and $R$. sibirica variants cocirculate in $R$. turanicus ticks. This data was confirmed by another study conducted by Song et al. (37) in the same country that indicates the occurrence of several SFG rickettsiae in $R h$. turanicus collected from several ruminants. Rickettsia massiliae DNA was previously found in the salivary glands, and saliva of $R h$. turanicus and its specific antibodies were also detected in patient sera. This may suggest, firmly, that $R h$. turanicus act as a potential vector and reservoir for this bacterium (38).

Furthermore, analysis of potential risk factors demonstrated three interesting facts related to geographic regions, potential tick 


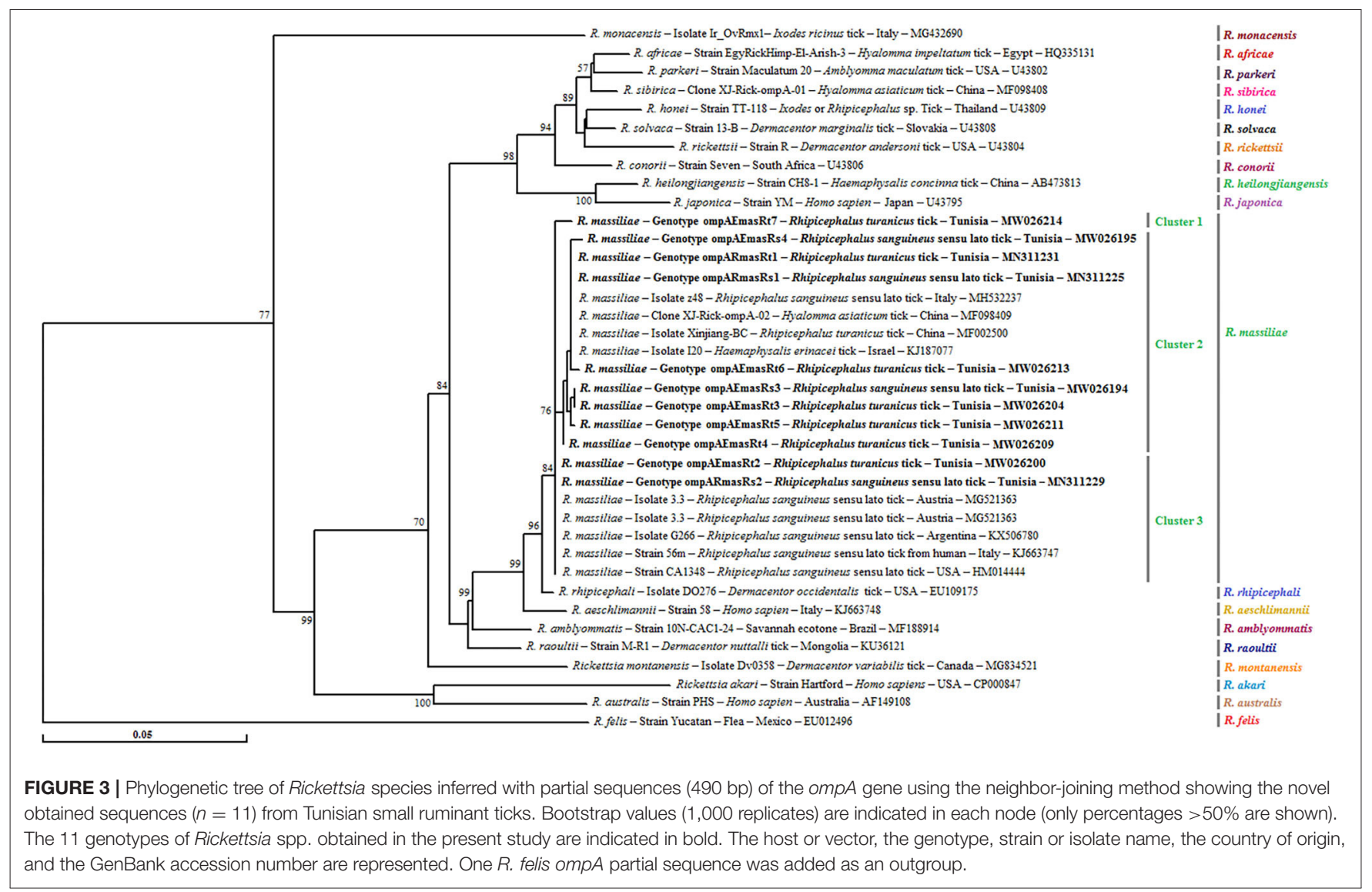

vector species, and infested hosts. Firstly, the positive rates of SFG Rickettsia in ticks were significantly higher in Beja (39\%) than in Bizerte (13.9\%) governorate. This discrepancy in prevalence rates according to geographic regions could be mainly explained by the diversity and heterogeneity of livestock population especially in El Alia locality and differences in husbandry practices, farm organization, wildlife reservoir hosts, and/or abiotic factors like the air temperature and the relative humidity that significantly affect the distribution of potential tick vectors. In addition, the higher rate of Rickettsia spp. observed in the governorate of Beja exclusively represented by the locality of Amdoun may be partly explained by the abundant presence in this region of $I$. ricinus considered to be one of the most important vectors of rickettsiae around the world (9). The infection of Rhipicephalus ticks with Rickettsia species may therefore come from infected small ruminants earlier infested with Rickettsia-positive I. ricinus ticks during wet seasons (9). Secondary, the positive rate in Rh. turanicus ticks (23.4\%) was significantly higher compared to $R$ h. sanguineus s.l. (9.5\%). This result is in line with those presented by Ghafar et al. (39) indicating a higher prevalence of $R$. massiliae and $R$. slovaca infections in $R h$. turanicus ticks from Pakistan compared to other tick species. Furthermore, risk factor analysis showed that ticks collected from goats (23.2\%) were more infected with Rickettsia spp. than those infesting sheep
(7.9\%) which is consistent with the same result of Ghafar et al. (39) in Pakistan.

In this study, R. massiliae was detected in Rh. turanicus and $R h$. sanguineus s.l., thus confirming its occurrence especially in the north of Tunisia. In our country, previous studies have reported the presence of $R$. massiliae in $R h$. sanguineus s.l. ticks collected from sheep situated in the center (6) and more recently in camels located in the center and the south (8). Similarly, $R$. massiliae has been also identified in Rh. turanicus and $R h$. sanguineus s.l. from Algeria, Italy, Cyprus, and Greece (15, 34, 40), in $R h$. sanguineus s.l. ticks from Morocco (41, 42), Spain, and Italy $(12,43)$, and in $R h$. turanicus ticks from China (36) and Pakistan (39).

In the present study, $R$. monacensis DNA was detected in $R$ h. sanguineus s.l. tick specimens removed from goats. These results consolidate previous data describing the presence of this bacterium in questing $I$. ricinus ticks (9), and in camels and their infesting $H$. impeltatum ticks (8). Besides, wide geographical distribution of this pathogen was noted particularly in the Mediterranean region (Italy and Spain) and from other countries like Costa Rica and Nicaragua (44-46). Interestingly, this species was identified as a zoonotic pathogen able to cause from moderate to severe illness in humans (19). The detection in Tunisia of $R$. monacensis DNA in $R h$. 


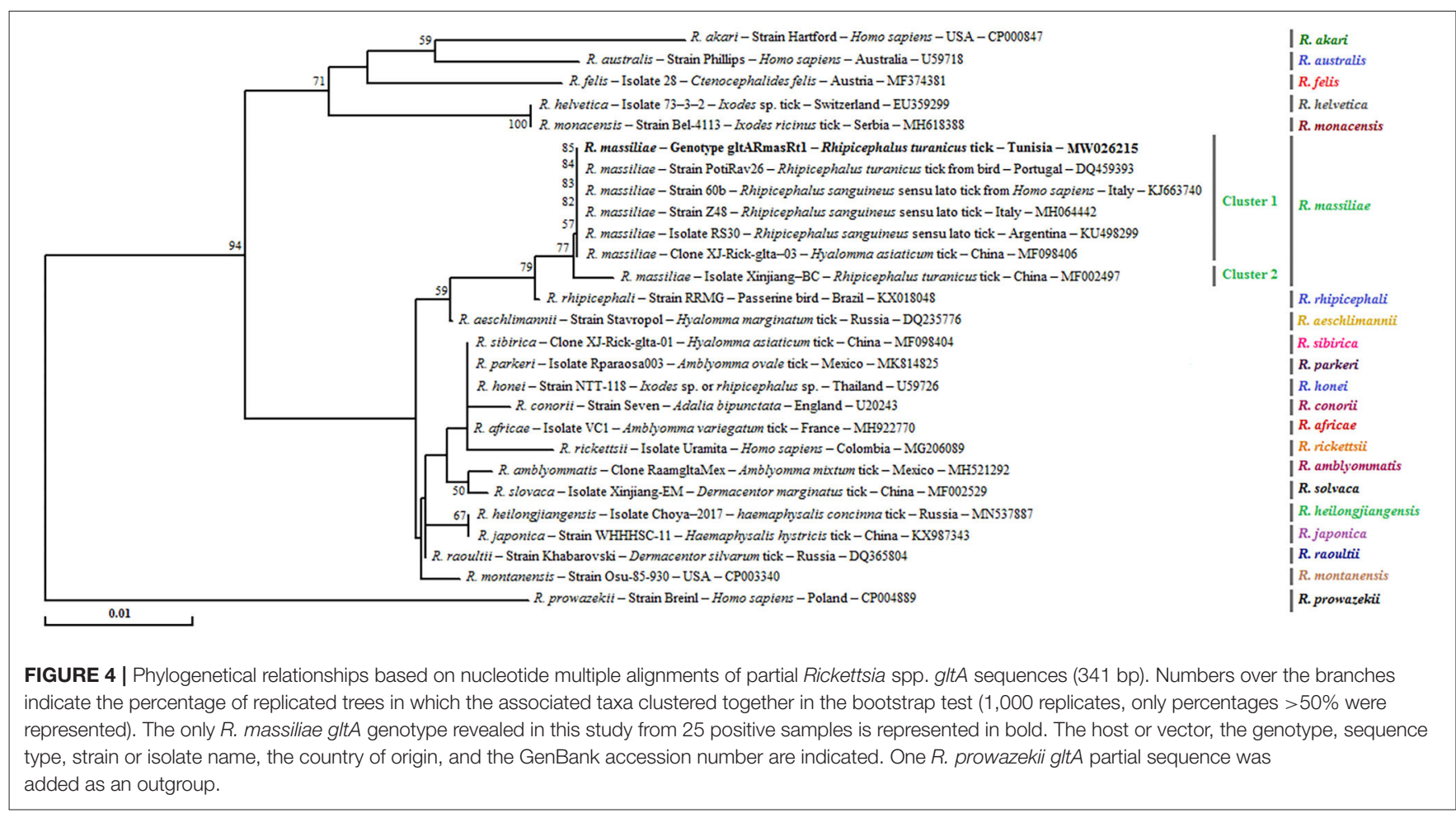

sanguineus s.l. ticks collected from goats suggests that, even if the circulation in the environment is essentially maintained by $I$. ricinus ticks, there may be other species incriminated in the transmission of this bacterial species as suggested in other reports from several countries $(19,47)$. Our findings highlight the need of extensive studies in the Rh. sanguineus s.l. tick complex collected from small ruminants and other domestic animals principally dogs to assess and predict the potential risks for humans.

However, given the growing occurrence of novel Rickettsia species with unidentified pathogenicity, it will be essential to carry out supplementary genetic characterization of the revealed Rickettsia spp. by using a combination of genetic markers such as $\operatorname{mp} p A$, and $g l t A$, in addition to the $\operatorname{omp} B$ gene. In the present study, phylogenetic trees based on the three gene fragments showed higher genetic diversity among the revealed $R$. massiliae isolates by using ompA and $\operatorname{omp} B$ genes compared to the gltA gene. This result is in line with those presented by Ereqat et al. (11) and Chisu et al. (48) investigating Palestinian and Sardinian ticks, respectively.

By analyzing ompB partial sequences, two genotypes (ompBRmasRs1 and ompBRmasRt1) infecting Rh. turanicus and $R h$. sanguineus s.l. tick specimens were found similar to that isolated from R. massiliae strain MTU5 (CP000683) recovered from $R h$. turanicus ticks collected on horses in Camargues, France (49), suggesting its potential spread in several Mediterranean countries. The remaining genotypes (ompBRmasRs2 and ompBRmasRt2) also infecting both tick species were found identical to $R$. massiliae Bar29
(AF123710) earlier identified in Rh. sanguineus s.l. ticks from Spain based on the same gene (50) and from Tunisia based on the 23S-5S intergenic spacer (6). Additionally, on the basis of the ompA phylogenetic tree, we found that $R$. massiliae isolated from Rhipicephalus ticks showed genetic divergence with novel genotypes, which indicates that these isolates infecting different tick species may come from various origins, hosts, and reservoirs. Thus, this finding needs to be further investigated.

Based on $\operatorname{omp} B$ phylogeny, low genetic diversity was observed among $R$. monacensis genotypes identified in this study. Indeed, one genotype (ompBRmonRs1) was found to be $100 \%$ similar to the corresponding sequence of $R$. monacensis strain $\mathrm{CN} 45 \mathrm{Kr}$ (EU883092) infecting a patient from South Korea (51), revealing its widespread distributions and potential risk for human. Thus, for a more accurate classification of our revealed $R$. monacensis isolates, further testing and phylogenetic analysis with additional genes are needed since no sequences of the two other genes isolated from this Rickettsia species were obtained in this study.

Therefore, the observation of these two zoonotic Rickettsia species, $R$. massiliae and $R$. monacensis, in investigated regions indicates a possible threat to resident humans. Indeed, infected tick species can also infest various domesticated animals and therefore constitute a possible risk for transmission of SFG rickettsiae to humans (3). However, the pathogenicity of this bacterium to humans is not well-understood (48). Consequently, supplementary trials are needed to investigate the pathogenicity of the revealed Rickettsia species and whether found tick species can transmit these pathogens in humans. 


\section{CONCLUSIONS}

The present study confirms the occurrence of human-pathogenic Rickettsia species in $R h$. sanguineus s.l. and $R h$. turanicus ticks collected from small ruminants in Tunisia. Our findings expand knowledge on ticks collected from domestic animals and highlight the range of infectious agents that may be transmitted by ticks to humans and animals.

\section{DATA AVAILABILITY STATEMENT}

The datasets presented in this study can be found in online repositories. The names of the repository/repositories and accession number(s) can be found in the article/supplementary material.

\section{ETHICS STATEMENT}

The animal study was reviewed and approved by The Ethics Committee of the National School of Veterinary Medicine of Sidi Thabet, University of Manouba. Written informed consent was obtained from the owners for the participation of their animals in this study.

\section{AUTHOR CONTRIBUTIONS}

$\mathrm{HB}$, LM, and MB conceived the idea. HB and MD-J carried out the fieldwork. HB, RS, and SZ performed the experiments.

\section{REFERENCES}

1. Parola P, Paddock CD, Socolovschi C, Labruna MB, Mediannikov O, Kernif $\mathrm{T}$, et al. Update on tick-borne rickettsioses around the world: a geographic approach. Clin Mic Rev. (2013) 26:657-702. doi: 10.1128/CMR.00 032-13

2. Abdad MY, Abou Abdallah R, Fournier PE, Stenos J, Vasoo S. A concise review of the epidemiology and diagnostics of rickettsioses: Rickettsia and Orientia spp. J Clin Mic. (2018) 56:1-12. doi: 10.1128/JCM.01728-17

3. Parola P, Paddock CD, Raoult D. Tick-borne rickettsioses around the world: emerging diseases challenging old concepts. Clinl Mic Rev. (2005) 18:719-56. doi: 10.1128/CMR.18.4.719-756.2005

4. Znazen A, Khrouf F, Elleuch N, Lahiani D, Marrekchi C, M'Ghirbi $\mathrm{Y}$, et al. Multispacer typing of Rickettsia isolates from humans and ticks in Tunisia revealing new genotypes. Parasit Vectors. (2013) 6:1-7. doi: 10.1186/1756-3305-6-367

5. Khrouf F, Sellami H, Elleuch E, Hattab Z, Ammari L, Khalfaoui M, et al. Molecular diagnosis of Rickettsia infection in patients from Tunisia. Ticks Tick Borne Dis. (2016) 7:653-6. doi: 10.1016/j.ttbdis.2016.02.010

6. Khrouf F, M'Ghirbi Y, Znazen A, Ben Jemaa M, Hammami A, Bouattour A. Detection of Rickettsia in Rhipicephalus sanguineus ticks and Ctenocephalides felis fleas from southeastern Tunisia by reverse line blot assay. J Clin Mic. (2014) 52:268-74. doi: 10.1128/JCM.01925-13

7. Demoncheaux JP, Socolovschi C, Davoust B, Haddad S, Raoult D, Parola P. First detection of Rickettsia aeschlimannii in Hyalomma dromedarii ticks from Tunisia. Ticks Tick Borne Dis. (2012) 3:398-402. doi: 10.1016/j.ttbdis.2012.10.003

8. Selmi R, Ben Said M, Ben Yahia H, Abdelaali H, Messadi L. Molecular epidemiology and phylogeny of spotted fever group Rickettsia in camels (Camelus dromedarius) and their infesting ticks from Tunisia. Transbound Emerg Dis. (2020) 67:733-44. doi: 10.1111/tbed.13392
$\mathrm{HB}$ and $\mathrm{MB}$ performed risk factor analysis, genotyping, and phylogenetic study. $\mathrm{HB}$ and $\mathrm{MB}$ wrote the manuscript and $\mathrm{HB}$, RS, LM, and MB finalized it. All authors read and approved the final version.

\section{FUNDING}

This work was supported by the research laboratory Laboratoire d'épidémiologie d'infections enzootiques des herbivores en Tunisie (LR01AGR16) and the research projects Screening and molecular characterization of pathogenic and zoonotic bacteria of medical and economic interest in cattle and camel ticks in Tunisia (19PEJC07-22) and Study of the bacterial microbiota in ticks with a medical and economic impact in Tunisia: contribution to the control of vector-borne bacterial diseases (P2ES2020-D4P1), all of which are funded by the Ministry of Higher Education and Scientific Research of Tunisia.

\section{ACKNOWLEDGMENTS}

The authors are thankful to Drs. Leilla Sayeh, Hichem Talhaoui, Rabeh Bouazizi, and Samir Touil, and they acknowledge their technicians for their help and their contribution in facilitating the access to the farms. The authors would like to thank Ms. Khaoula Khraifi and Ms. Imen Nasralli for their help in performing the experiments.

9. Sfar N, M'Ghirbi Y, Letaief A, Parola P, Bouattour A, Raoult D. First report of Rickettsia monacensis and Rickettsia helvetica from Tunisia. An Trop Med Parasitol. (2008) 102:561-4. doi: 10.1179/136485908X311795

10. Beati L, Raoult D. Rickettsia massiliae sp. nov., a new spotted fever group Rickettsia. Int J Syst Bacteriol. (1993) 43:839-40. doi: 10.1099/00207713-43-4-839

11. Ereqat S, Nasereddin A, Al-Jawabreh A, Azmi K, Harrus S, Mumcuoglu K. Molecular detection and identification of spotted fever group Rickettsiae in ticks collected from the West Bank, Palestinian territories. PLoS Neg Trop Dis. (2016) 10:e0004348. doi: 10.1371/journal.pntd.0004348

12. Chisu V, Masala G, Foxi C, Socolovschi C, Raoult D, Parola P. Rickettsia conorii israelensis in Rhipicephalus sanguineus ticks, Sardinia, Italy. Ticks Tick Borne Dis. (2014) 5:446-8. doi: 10.1016/j.ttbdis.2014.02.003

13. Babalis T, Tselentis Y, Roux V, Psaroulaki A, Raoult D. Isolation and identification of a rickettsial strain related to Rickettsia massiliae in Greek ticks. Am J Trop Med Hyg. (1994) 50:365-72. doi: 10.4269/ajtmh.1994.50.365

14. Cardeñosa N, Segura F, Raoult D. Serosurvey among Mediterranean spotted fever patients of a new spotted fever group rickettsial strain (Bar29). Eur J Epidemiol. (2003) 18:351-6. doi: 10.1023/A:1023654400796

15. Mura A, Masala G, Tola S, Satta G, Fois F, Piras P, et al. First direct detection of rickettsial pathogens and a new rickettsia, 'Candidatus Rickettsia barbariae', in ticks from Sardinia, Italy. Clin Mic Infect. (2008) 14:1028-33. doi: 10.1111/j.1469-0691.2008.02082.x

16. Fernández de Mera I, Zivkovic Z, Bolaños M, Carranza C, Pérez-Arellano J, Gutiérrez C, et al. Rickettsia massiliae in the Canary Islands. Emerg Infect Dis. (2009) 15:1869-70. doi: 10.3201/eid1511.090681

17. Vitale G, Mansueto S, Rolain J, Raoult D. Rickettsia massiliae human isolation. Emerg Infect Dis. (2006) 12:174-5. doi: 10.3201/eid1201.050850

18. Garcia-Garcia JC, Portillo A, Nunez MJ, Santibanez S, Castro B, Oteo JA. A patient from Argentina infected with Rickettsia massiliae. Am J Trop Med Hyg. (2010) 82:691-2. doi: 10.4269/ajtmh.2010.09-0662 
19. Madeddu G, Mancini F, Caddeo A, Ciervo A, Babudieri S, Maida I, et al. Rickettsia monacensis as cause of Mediterranean spotted fever-like illness, Italy. Emerg Infect Dis. (2012) 18:702-4. doi: 10.3201/eid1804.1 11583

20. Battisti E, Zanet S, Boraso F, Minniti D, Giacometti M, Duscher G, et al. Survey on tick-borne pathogens in ticks removed from humans in Northwestern Italy. Vet Parasitol Reg Stud Rep. (2019) 6:112-6. doi: 10.1016/j.vprsr.2019.100352

21. Walker A, Bouattour A, Camicas J, Estrada-Peña A, Horak I, Latif A, et al. Ticks of Domestic Animals in Africa: A Guide to Identification of Species The University of Edinburgh. Bioscience Reports: Edinburgh (2013). p. 233-68.

22. Black WC, Piesman J. Phylogeny of hard- and soft-tick taxa (Acari: Ixodida) based on mitochondrial 16S rDNA sequences. Proc Natl Acad Sci USA. (1994) 91:10034-8. doi: 10.1073/pnas.91.21.10034

23. Choi YJ, Jang WJ, Ryu JS, Lee SH, Park KH, Paik HS, et al. Spotted fever group and typhus group rickettsioses in humans, South Korea. Emerg Infect Dis. (2005) 11:237-44. doi: 10.3201/eid1102.040603

24. Oteo JA, Portillo A, Santibáñez S, Blanco JR, Pérez-Martínez L, Ibarra V. Cluster of cases of human Rickettsia felis infection from Southern Europe (Spain) diagnosed by PCR. J Clin Mic. (2006) 44:2669-71. doi: 10.1128/JCM.00366-06

25. Regnery R, Olson J, Perkins B, Bibb W. Serological response to Rochalimaea henselae antigen in suspected cat-scratch disease. Lancet Infect Dis. (1992) 339:1443-5. doi: 10.1016/0140-6736(92)92032-B

26. Thompson JD, Higgins DG, Gibson TJ. Clustal W: improving the sensitivity of progressive multiple sequence alignment through sequence weighting position specific gab penalties and weight matrix choice. Nucleic Acids Res. (1994) 22:4673-80. doi: 10.1093/nar/22.22.4673

27. Tamura K, Nei M. Estimation of the number of nucleotide substitutions in the control region of mitochondrial DNA in humans and chimpanzees. Mol Biol Evol. (1993) 10:512-26.

28. Saitou N, Nei M. The neighbor-joining method: a new method for reconstructing phylogenetic trees. Mol Bio Evol. (1987) 4:406-25.

29. Felsenstein J. Confidence limits on phylogenies: an approach using the bootstrap. Evolution. (1985) 39:783-91. doi: 10.1111/j.1558-5646.1985.tb00420.x

30. Kernif T, Socolovschi C, Bitam I, Raoult D, Parola P. Vector-borne rickettsioses in North Africa. Infect Dis Clin North Am. (2012) 26:455-78. doi: 10.1016/j.idc.2012.03.007

31. Abdelkadir K, Palomar A, Portillo A, Oteo J, Ait-Oudhia K, Khelef D. Presence of Rickettsia aeschlimannii, 'Candidatus Rickettsia barbariae' and Coxiella burnetii in ticks from livestock in Northwestern Algeria. Ticks Tick Borne Dis. (2019)10:924-8. doi: 10.1016/j.ttbdis.2019.04.018

32. Belkahia H, Ben Said M, Ghribi R, Selmi R, Ben Asker A, Yahiaoui $\mathrm{M}$, et al. Molecular detection, genotyping and phylogeny of Anaplasma spp. in Rhipicephalus ticks from Tunisia. Acta Trop. (2019) 191:38-49. doi: 10.1016/j.actatropica.2018.12.034

33. Ben Said M, Belkahia H, Alberti A, Zobba R, Bousrih M, Yahiaoui M, et al. Molecular survey of anaplasma species in small ruminants reveals the presence of novel strains closely related to A. phagocytophilum in Tunisia. Vector Borne Zoonotic Dis. (2015) 15:580-90. doi: 10.1089/vbz.2015.1796

34. Psaroulaki A, Spyridaki I, Ioannidis A, Babalis T, Gikas A, Tselentis Y. First isolation and identification of Rickettsia conorii from ticks collected in the region of Fokida in Central Greece. J Clin Mic. (2003) 41:3317-9. doi: 10.1128/JCM.41.7.3317-3319.2003

35. Germanakis A, Chochlakis D, Angelakis E, Tselentis Y, Psaroulaki A. Rickettsia aeschlimannii infection in a man, Greece. Emerg Infect Dis. (2013) 19:1176-7. doi: 10.3201/eid1907.130232

36. Wei Q, Guo L, Wang A, Mu L, Zhang K, Chen C, et al. The first detection of Rickettsia aeschlimannii and Rickettsia massiliae in Rhipicephalus turanicus ticks, in northwest China. Parasit Vectors. (2015) 10:631-41. doi: 10.1186/s13071-015-1242-2
37. Song S, Chen C, Yang M, Zhao S, Wang B, Hornok S, et al. Diversity of Rickettsia species in border regions of northwestern China. Parasit Vectors. (2018) 13:634-41. doi: 10.1186/s13071-018-3233-6

38. Matsumoto K, Ogawa M, Brouqui P, Raoult D, Parola P. Transmission of Rickettsia massiliae in the tick, Rhipicephalus turanicus. Med Vet Entomol. (2005) 19:263-70. doi: 10.1111/j.1365-2915.2005.00569.x

39. Ghafar A, Khan A, Cabezas-Cruz A, Gauci C, Niaz S, Ayaz S, et al. An assessment of the molecular diversity of ticks and tick-borne microorganisms of small ruminants in Pakistan. Microorganisms. (2020) 8:1428-31. doi: 10.3390/microorganisms 8091428

40. Chochlakis D, Ioannou I, Sandalakis V, Dimitriou T, Kassinis N, Papadopoulos B, et al. Spotted fever group Rickettsiae in ticks in Cyprus. Microbial Ecol. (2012) 63:314-23. doi: 10.1007/s00248-011-9926-4

41. Boudebouch N, Sarih M, Socolovschi C, Fatihi T, Chakib A, Amarouch H, et al. Spotted fever group rickettsioses documented in Morocco. Clin Mic Infect. (2009) 15(Suppl. 2):257-8. doi: 10.1111/j.1469-0691.2008.02276.x

42. Sarih M, Socolovschi C, Boudebouch N, Hassar M, Raoult D, Parola P. Spotted fever group rickettsiae in ticks, Morocco. Emerg Infect Dis. (2008) 14:1067-73. doi: 10.3201/eid1407.070096

43. Márquez FJ, Rodríguez-Liébana JJ, Soriguer RC, Muniaín MA, BernabeuWittel M, Caruz A, et al. Spotted fever group Rickettsia in brown dog ticks Rhipicephalus sanguineus in southwestern Spain. Parasitol Res. (2008) 103:119-22. doi: 10.1007/s00436-008-0938-Z

44. Springer A, Montenegro V, Schicht S, Wölfel S, Schaper S, Chitimia-Dobler L, et al. Detection of Rickettsia monacensis and Rickettsia amblyommatis in ticks collected from dogs in Costa Rica and Nicaragua. Ticks Tick Borne Dis. (2018) 9:1565-72. doi: 10.1016/j.ttbdis.2018.08.002

45. Estrada-Peña A, Roura X, Sainz A, Miró G, Solano-Gallego L. Species of ticks and carried pathogens in owned dogs in Spain: results of a one-year national survey. Ticks Tick Borne Dis. (2017) 8:443-52. doi: 10.1016/j.ttbdis.2017.02.001

46. Pennisi M, Persichetti M, Serrano L, Altet L, Reale S, Gulotta L, et al. Ticks and associated pathogens collected from cats in Sicily and Calabria (Italy). Parasit Vectors. (2015) 7:512-20. doi: 10.1186/s13071-015-1128-3

47. Pascucci I, Di Domenico M, Curini V, Cocco A, Averaimo D, D'Alterio $\mathrm{N}$, et al. Diversity of Rickettsia in ticks collected in Abruzzi and Molise regions (central Italy). Microorganisms. (2019) 13:696-71. doi: 10.3390/microorganisms7120696

48. Chisu V, Foxi C, Mannu R, Satta G, Masala G. A five-year survey of tick species and identification of tick-borne bacteria in Sardinia, Italy. Ticks Tick Borne Dis. (2018) 9:678-81. doi: 10.1016/j.ttbdis.2018.02.008

49. Blanc G, Ogata H, Robert C, Audic S, Claverie J, Raoult D. Lateral gene transfer between obligate intracellular bacteria: evidence from the Rickettsia massiliae genome. Genome Res. (2007) 17:1657-64. doi: 10.1101/gr.6742107

50. Roux V, Raoult D. Phylogenetic analysis of members of the genus Rickettsia using the gene encoding the outer-membrane protein $\mathrm{rOmpB}$ (ompB). Int J Syst Evol Mic. (2000) 50:1449-55. doi: 10.1099/00207713-50-4-1449

51. Kim Y, Choi Y, Lee K, Ahn K, Kim H, Klein T, et al. First isolation of Rickettsia monacensis from a patient in South Korea. Mic Immunol. (2017) 61:258-63. doi: 10.1111/1348-0421.12496

Conflict of Interest: The authors declare that the research was conducted in the absence of any commercial or financial relationships that could be construed as a potential conflict of interest.

Copyright (c) 2021 Belkahia, Selmi, Zamiti, Daaloul-Jedidi, Messadi and Ben Said. This is an open-access article distributed under the terms of the Creative Commons Attribution License (CC BY). The use, distribution or reproduction in other forums is permitted, provided the original author(s) and the copyright owner(s) are credited and that the original publication in this journal is cited, in accordance with accepted academic practice. No use, distribution or reproduction is permitted which does not comply with these terms. 